\title{
Dealing with non-normality: An introduction and step-by-step guide using $R$
}

\author{
Matthew G. R. Courtney \\ Assessment Research Centre, Melbourne Graduate School of Education, The University of Melbourne, Victoria, \\ Australia \\ e-mail: matthew.courtney@unimelb.edu.au
}

Kevin C. Chang

Statistics Consulting Centre, Department of Statistics, The University of Auckland, Auckland, New Zealand e-mail: k.chang@auckland.ac.nz

\begin{abstract}
Having spent much time tutoring and teaching introductory statistics courses for education and social science majors and supporting students embarking on statistical work, we have noticed that students often wonder why one would normalize data and how to go about it. This article presents some simple notes on the why and the how, then illustrates these procedures via some introductory arithmetic and graphing commands in R. The paper finishes by guiding students to use a Shiny app to automatically normalize multiple variables in a larger dataset. Real data on student Internet behavior is used and correlations between variables of interest are compared before and after transformations.
\end{abstract}

Keywords:

Teaching; statistics; non-normality; skewness; kurtosis; power; transformation; Box-Cox lambda

\section{Introduction}

For many statistical procedures, it is assumed that variables generally follow a normal distribution, sometimes called the 'bell curve'. If we are interested in detecting relationships between variables, deviations from the bell curve can distort results. The more distorted the distribution, the higher the chances can be of committing either a Type I error (incorrect rejection of a true null hypothesis) or Type II error (incorrectly retaining a null hypothesis). For example, it is well documented the correlation between two variables may be a poor measure of association when data is not normal (Kowalski, 1972). For these reasons, assessments and adjustments for non-normality can represent an important part of data preparation for any statistical analysis in a student project.

\section{Assessments of (non)-normality}

Deviations from normal distribution are generally assessed by way of two criteria: skewness and kurtosis. Here we consider only unimodal continuous distributions. A variable can be either positively-skewed, not skewed, or negatively-skewed. Positively-skewed variables have a longer tail to the right and therefore often (but not always) an off-peak mean to the right (the positive side; considered positively skewed). Normally distributed variables are symmetrical and therefore have a mean which is at the peak of the curve (not skewed). Negatively-skewed

This is the author manuscript accepted for publication and has undergone full peer review but has not been through the copyediting, typesetting, pagination and proofreading process, which may lead to differences between this version and the Version of Record. Please cite this article as doi: $10.1111 /$ test.12154

This article is protected by copyright. All rights reserved. 
variables have a longer tail to the left and therefore often (but not always) an off-peak mean to the left (the negative side; considered negatively skewed). Figure 1 gives some simple illustrations of these three forms of skewness.

\section{Figure 1. Three Forms of Skewness}

Whilst skewness is a measure of the balance of the tails in a distribution, kurtosis is a measure of the slenderness (or broadness) of the distribution. A distribution that looks like a tall and slender mountain toward the top, but with long flat feet (tails), was long ago called leptokurtic (lepto means slender; more kurtosis than normal). A distribution that appears like a bell curve is defined as mesokurtic (normal), whilst a distribution that looks like a broad mountain, with short light feet (tails), was long ago called platykurtic (platy means broad; less kurtosis than normal). Thanks to the statistician William Sealy Gosset (aka, "Student"; Student, 1927, p. 160), one useful way to remember the platykurtic and leptokurtic distributions is to visualize the broad but short-tailed platypus and two kangaroos leaping and (or "lepping") toegether (Figure 2).

\section{Figure 2. Platykurtic and Leptokurtic Distributions}

Adapted from "Errors of Routine Analysis," by Student, 1927, Biometrika, 19(1/2), p. 160.

It must be noted that distributions must be scaled and located in the same way to be able to 'look at' their kurtosis. There are many different measures which can be used to represent skewness and kurtosis of a distribution. In this paper, we will use the standardized third central moment as the measure of skewness, and the standardized fourth central moment as the measure of kurtosis. For both of these measures, distributions being compared with respect to skewness and kurtosis are standardized by having the same mean and the same standard deviation.

\section{Commonly used transformative exponents}

Traditionally, that is prior to the onset of modern computing, when variables deviated greatly from normality, if statisticians wanted to make data more 'normal', they often applied a mathematical function (generally some type of power) to a variable in an attempt to make it more 'normally' distributed. Table 1 presents a selection of exponents traditionally used to normalize data (see Osbourne, 2010, for full review). As you might have picked up, each of the seven transformations (other than None) in the Table 1 represent a class of transformations called power transformations. The Greek symbol $\lambda$ (lambda) is often used to represent the power (called exponent). Another very important transformation is the log transformation; for mathematical reasons not considered here, this corresponds to lambda $=0$. 


\begin{tabular}{lcc}
\multicolumn{2}{l}{ Table } & 1. Traditionally used exponents for transformations \\
\hline $\boldsymbol{\lambda}$ & $\mathrm{Y}$ & \multicolumn{2}{c}{ Description of transformation } \\
\hline 3 & $\mathrm{Y}^{3}$ & Cubed \\
2 & $\mathrm{Y}^{2}$ & Quadratic \\
1 & $\mathrm{Y}$ & None \\
0.5 & $\sqrt{\mathrm{Y}}$ & Square root \\
-0.5 & $1 / \sqrt{\mathrm{Y}}$ & Reciprocal (inverse) square root \\
-1 & $1 / \mathrm{Y}$ & Reciprocal (inverse) \\
-2 & $1 / \mathrm{Y}^{2}$ & Reciprocal (inverse) \\
\hline
\end{tabular}

\section{The usefulness of exponentiation for skewed variables}

Let us consider for a moment the general effects of applying a couple of the power functions (mentioned in Table 1) to variables exhibiting different forms of skewness. For illustration purposes, we consider two small datasets, and comment that such sample sizes are generally considered to be too small for reliable assessment of non-normality and transformations.

After installing and loading the psych and normalr packages in $\mathrm{R}$, we can create the following simple vector (variable1) and identify its level of skewness and kurtosis:

variable1 <- $c(1,5,9,9,9,9,10,10,10,11,11,12)$

It is important to first check the distribution of a variable visually. One helpful way to do this is by using the quantile-quantile (QQ) plot (qqnorm). This plots the sample data against a normal distribution. In addition to this, the qqline function plots the line that would be produced if sample data were normal. This helps us to visualise how far a variable deviates from normality.

qqnorm(variable1)

qqline (variable1)

The QQ plots can also be supplemented by statistical estimates of skewness and kurtosis. As noted above, in this paper we use the standardized third and fourth central moments as measures of skewness and kurtosis. And 3 is subtracted from the standardized fourth central moment because this is its value for the normal distribution. For example, Hair, Black, Babin, and Anderson (2010) suggest that skewness values falling outside |1| indicate substantially skewed distribution.

skew(variable1)

kurtosi(variable1)

\#skewness is -1.47 (negative skewness) 
\#kurtosis is 1.19 (slightly leptokurtic)

The QQ Plot for variable1 is presented on the left-hand side of Figure 3. We note large deviation off the QQ line at the bottom of the plot, suggestive of non-normality.

\section{Figure 3. QQPlot of variable1 and variable1. Exp3}

In an attempt to resolve the issue, we might try applying a power function to each of the values. Let us consider what happens when we raise each of the values in the variable to the power of 3 :

variable1.Exp3 <- variable1^3

variable1. Exp3

\#values now: 1, 125, 729, 729, 729, 729, 1000, 1000, 1000, 1331, 1331, 1728

qqnorm (variable1.Exp3)

qqline (variable1.Exp3)

skew(variable1. Exp3)

kurtosi(variable1.Exp3)

This transformation is illustrated by the two QQ plots in Figure 3. We can see that the application of the cube function (i.e., to the power of 3) to each value tends to increase the larger values more than the smaller values. Therefore, this function disproportionately broadens the right (positive) side of the distribution ultimately creating a more normally distributed pattern of data. The QQ plot on the right (Figure 3, variable1. Exp3) illustrates a more normal distribution with points in the graph closer to the normal line. The resultant skewness and kurtosis values of -0.18 and -0.75 , respectively, also suggest a more normal distribution.

The following variable represents the opposite problem, positive skewness:

variable2 <- $c(1,2,2,3,3,3,4,4,4,4,8,12)$

qqnorm(variable2)

qqline (variable2)

skew(variable2)

kurtosi(variable2)

Interpretation of the QQ plot (Figure 4, variable2) suggests that variable2 is moderately positively skewed and leptokurtic. The skewness and kurtosis measures support this interpretation with values estimated at 1.47 and 1.19, respectively. In an attempt to resolve this issue, we might try applying an inverse function, namely, lambda $=-0.1$. This power function is 
now detailed for $\mathrm{x}_{1}(1.00)$ and $\mathrm{x}_{\mathrm{n}}(12.00)$, respectively (i.e., the first then twelfth value of the variable2).

$$
\begin{aligned}
& \text { For } X_{1}: 1.00^{-0.1}=\left(\frac{1.00}{1}\right)^{\frac{-1}{10}}=\left(\frac{1}{1.00}\right)^{\frac{1}{10}}=\left(\sqrt[10]{\frac{1}{1.00}}\right)^{1}=(1.00)^{1}=1.00 \\
& \text { For } X_{n}: 12.00^{-0.1}=\left(\frac{12.00}{1}\right)^{\frac{-1}{10}}=\left(\frac{1}{12.00}\right)^{\frac{1}{10}}=\left(\sqrt[10]{\frac{1}{12.00}}\right)^{1}=(0.78)^{1}=0.78
\end{aligned}
$$

We can apply the exponent, -0.1 , to variable2 in the R program as follows:

variable2.ExpNeg@.1<- variable2^ $-\odot .1$

variable2. ExpNeg๑.1

\#values now (2dp):1,125, 729, 729, 729, 729, 1000, 1000, 100๑, 1331, 1331, 1728

qqnorm (variable2. ExpNeg@ .1)

qqline (variable2. ExpNeg॰.1)

skew (variable2. ExpNeg@.1)

kurtosi(variable2. ExpNeg@.1)

\section{Figure 4. QQPlot of variable1 and variable1. Exp3}

There appears to be some improvement in the QQ plot for variable2 . Expneg 1.1 (Figure 4) with values more closely aligned with the normal line. Skewness (.05) and kurtosis $(-0.30)$ also appears to be less severe in absolute terms.

To illustrate the transformations taking place to variable2, Figure 5 (top) provides a series of histograms. The transformation from variable2 to variable2. ExpNeg $\odot .1$ is illustrated by the top left then top right histograms the figure: a. original to $b$. lambda $=-0.1$ applied.

\section{Figure 5. Application of lambda -0.1, reflection, and re-anchoring of positively skewed variable \\ Note. See notes in R script lines 163-171 for creation of the four histograms.}

Astute students will pick up on the fact that the transformed series (top right) now descends from 1.00 to 0.78 (whereas before it ascended from 1 to 12). If this were not dealt with, the variable would now incorrectly assign higher values to observations originally associated with lower values (and vice versa). To deal with this issue, we can simply reflect the variable by multiplying it by -1 (see Figure 5, c. reflected). 
variable2.reflected <- variable2.ExpNeg०.1* -1

variable2, reflected

\#values now(2dp):-1.00,-0.933,-0.933,-0.90,-0.90,-0.90,-0.87,-\#0.87,-0.87,-

$0.87,-\# \odot .81,-0.78$

As a result of the reflection, the values range from -1.00 to -0.78 . For the variable to revert to its original positive state, we can add a constant, in this case 2.00 , to peg the smallest value $\left(\mathrm{X}_{1}=-\right.$ $1.00)$ at 1.00 , in line with the variable's original minimum.

variable2.pegged <- reflected +2

variable2. pegged

\#values now(2dp):

$\# 1.00,1.07,1.07,1.10,1.10,1.10,1.13,1.13,1.13,1.13,1.19,1.22$

The application of the constant results in the variable illustrated in Figure 5, d. pegged.

\section{Issues of Scale}

A quick look at the $\mathrm{X}$ axes on the four graphs in Figure 5 tells us that for each graph, the variable's range changes. Although the final pegged variable starts at 1.00 (Figure 5, d. pegged), its maximum value reaches only 1.25 , a value well below the variable's original maximum of 12.00. This change of scale may be an issue if one would like to report transformed values with some sense of meaning (in line with the min and max values in the original scale). One way to resolve this issue is to apply the following rescaling formula:

$$
\left(\frac{x_{n}-x_{1}}{X_{n}-X_{1}}\right)\left(X-X_{1}\right)+x_{1}=X_{m}
$$

where, $x_{1}=$ original min value, $x_{n}=$ original max value, $X_{1}=$ transformed min value, $X_{n}=$ transformed max value, $X=$ transformed value to be scaled, $X_{m}=$ transformed value mapped into original range.

Let's consider the following variable transformation undertaken earlier in the paper:

the variable,

$$
\begin{aligned}
& 1,5,9,9,9,9,10,10,10,11,11,12 \\
& \text { via } X^{3} \text {, became, }
\end{aligned}
$$

1, 125, 729, 729, 729, 729, 1000, 1000, 1000, 1331, 1331, 1728

Let's look at mapping the $\mathbf{7 2 9}$ value back within its original min-max via the scaling formula: 


$$
\left(\frac{12-1}{1728-1}\right)(729-1)+1=5.64
$$

In R, this mapping procedure could be run for the whole variable as follows:

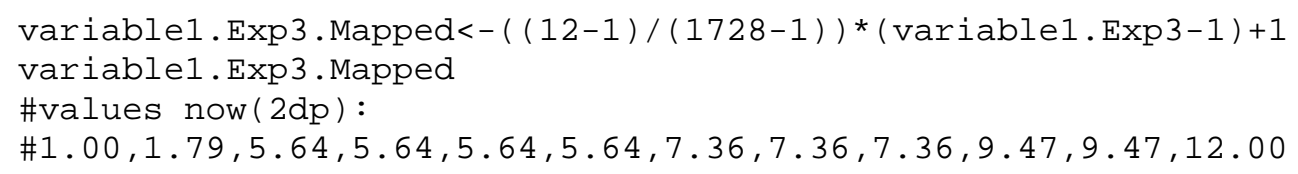

\section{Anchoring variables at 1.00}

As the very astute student may have noted, the two given example variables just so happened to be both initially anchored at $1.00\left(\mathrm{X}_{1}=1\right)$. This was done for a reason. To understand why, let's consider the application of $\lambda=3$ to the following variable that happens to be anchored at 0.25 .

$$
0.25,0.5,0.75,1,5,9,9,9,9,10,10,10
$$

Raising each value to the power of 3 would generate the following:

$$
\mathbf{0 . 0 2}, \mathbf{0 . 1 2 5}, \mathbf{0 . 4 2}, 1, \underline{125}, \underline{729}, \underline{729}, \underline{729}, \underline{729}, \underline{1000}, \underline{1000}, \underline{1000}
$$

As a result of this transformation, the first three values actually get smaller whilst the latter eight values get bigger. Clearly, exponents have incongruent effects on values under 1.00. By first applying a constant to anchor original variables at 1.00 , an exponent can be correctly identified and applied uniformly across all values in a variable (note that the constant could be a negative or positive value).

\section{Identifying the optimal exponent}

Of course in data analysis, we do not engage in an ongoing game of hit-and-miss to try to identify an exponent that provides an optimal level of skewness. This is where Box and Cox's (1964) paper comes into play. The transformation is:

$$
y(\lambda)=\left\{\begin{array}{ll}
\frac{y^{\lambda}-1}{\lambda}, & \text { if } \lambda \neq 0 \\
\log y, & \text { if } \lambda=0 .
\end{array}\right\}
$$

Statistical theory provides methods for estimating lambda from data. This is outside the scope of this paper, but, for the record, the method used in our Shiny app is called the Maximum Likelihood method. Whichever method used to estimate lambda, it is not a guarantee of optimal 
normality, and transformed data should be checked, but it has many practical and theoretical advantages.

\section{The normalr package}

Until now, students trying to normalize multiple variables in large-scale datasets would have to normalize each variable one at a time. For each variable, this would involve anchoring each variable, identifying the optimal exponent, then following through with the many steps identified above.

The many steps above are both cumbersome and make errors more likely to occur. This is where the normalr package provides an efficient way forward. The package extends previous work by Venables and Ripley (2002) who created the mass package to identify the optimal transformative exponent. Using the rules and protocols established in this paper, the normalr package quickly, accurately, and automatically normalizes all continuous variables in large datasets and maps them back within their original min and max values (Chang \& Courtney, 2017). For this paper, we focus on the following two functions in the R package: get Lambda, and normaliseData.

For illustrative purposes, we make use of an example dataset of four variables (958 cases) concerned with the self-reported Internet and social media habits of university students across Australia and New Zealand (Net . Habits . csv; supplementary materials). From left to right, the variables are NetUse (per day), TFBF (total Facebook friends), TAFBF (total actual FB friends), TFBFOC (total FB friends on campus). The word "actual friend" was defined as someone that the students know and interact with on a personal level. Note that all participants gave informed consent and ethical approval was granted by the institutional ethics committee.

As researchers we might be interested in the correlation between time spent online and the different friendship counts on Facebook. After reading in the dataset, we could run bivariate correlations between the following variables in $\mathrm{R}$ :

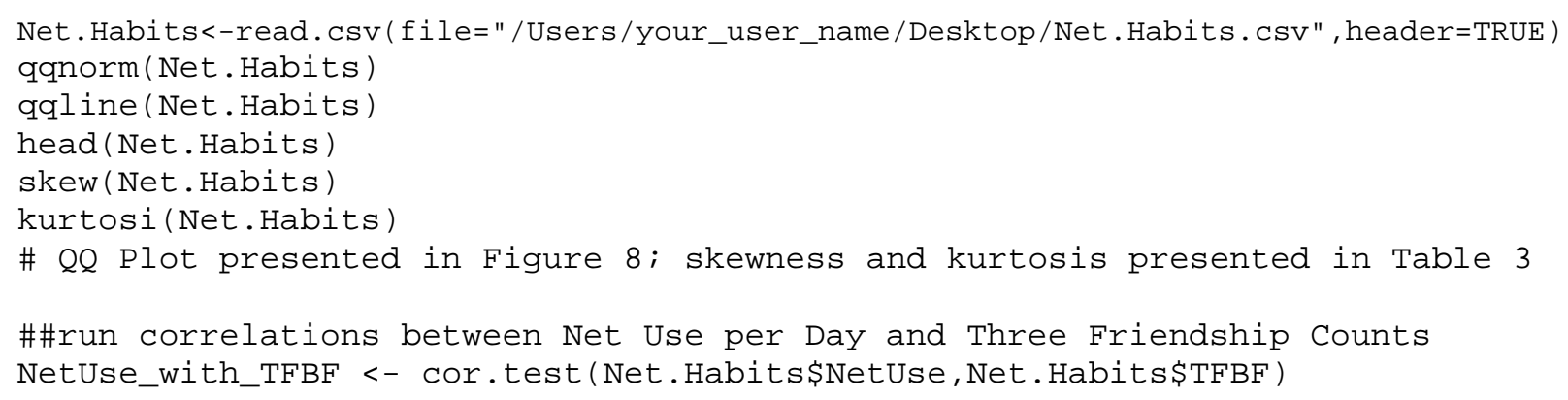




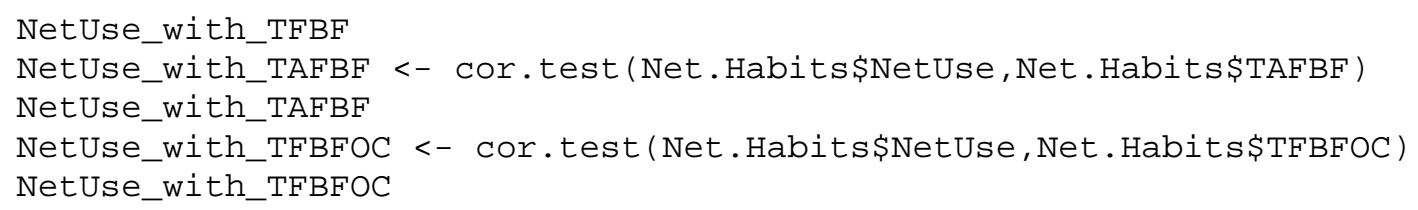

The three correlations run above are presented in the first row of Table 2 (Before normalisation).

Table 2.

Correlation Coefficients (Pearson) with NetUse Before and After Normalisation

\begin{tabular}{lccc}
\hline Dataset & Total FB Friends & Tot. Act. FB Friends & Tot. FB Friends on Campus \\
\hline Before transformation & $.14^{* * *}$ & $.17^{* * *}$ & $.13^{* * *}$ \\
After transformation & $.12^{* * *}$ & $.13^{* * *}$ & $.16^{* * *}$ \\
\hline
\end{tabular}

Note. $* p<.05, * * p<.01, * * * p<.001$.

It seems that all correlations between NetUse and Facebook friendship counts are positive, small, and statistically significant. To check that none of these results are an consequence of the variable being non-normal, we can transform the data and double-check. First we need to identify the optimal lambda values for each of the four variables by running the following code:

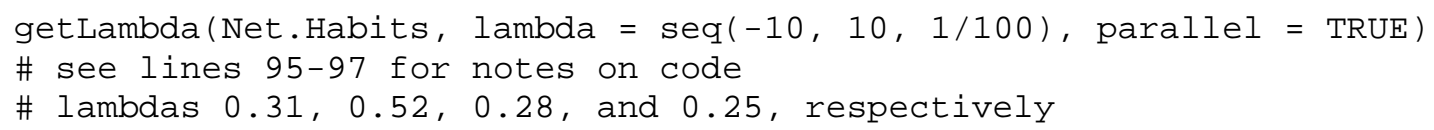

We can then normalise the four variables (as described previously in this paper) by applying the following script, then outputting transformed dataset to the desktop:

normaliseData(LambdaZeroV9, getLambda(LambdaZeroV9, parallel = TRUE))

\# see lines 100-107 for full instructions in Mac/Windows

We have also created a user-friendly online Shiny interface if you wanted to normalise data online. The following steps allow users to easily upload, transform, and download their files in a few easy mouse clicks:

Step 1: Go to the following Shiny App Address: https://kcha193.shinyapps.io/normalr/ Step 2: Choose your csv file by clicking on Browse (be sure to check/uncheck Header and choose comma separator as appropriate). This will generate the image in Figure 6.

Figure 6. Image of Upload of the LambdaZeroV9.csv File 
Step 3: Click Transformed Data to View the output (Figure 7).

\section{Figure 7. Image of transformed dataset}

Step 4: Click on the Download button to save your transformed file to your desktop (exported dataset will include headers by default). Change headers from default V1-V4 to NetUse TFBFOC and save as Net.Habits.Normal.csv. Using this dataset, we can run the same four correlations again:

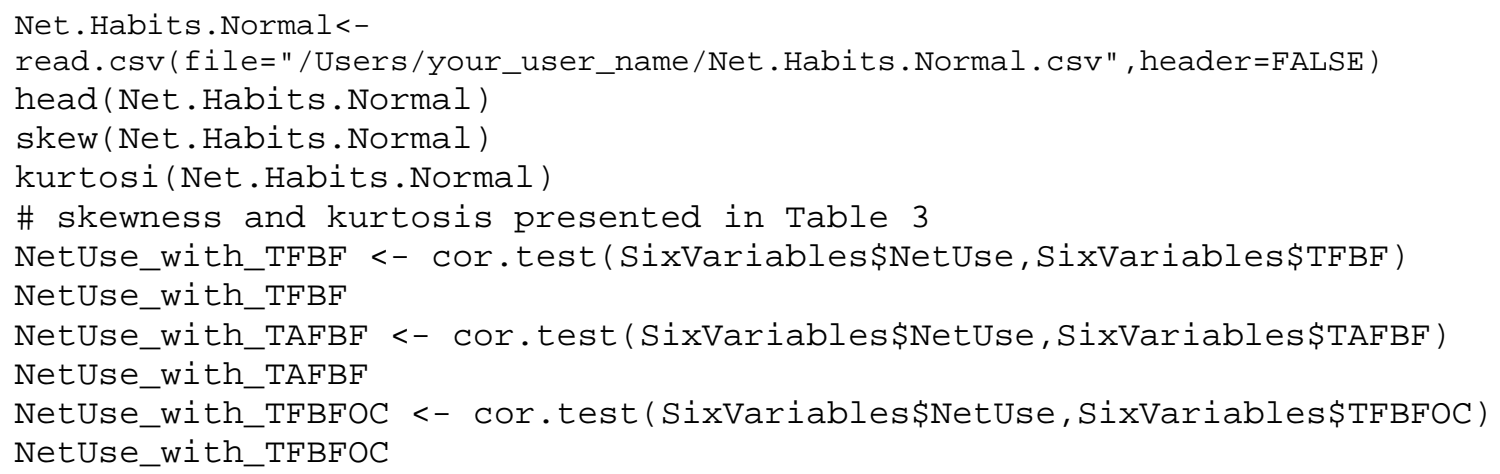

\section{Results of above transformations}

The procedures carried out with the normalr package (and Shiny app) function to normalize the variables in Net. Habits. csv dataset in an efficient way. As an example, Figure 8 presents the QQ plots for the TFBFOC variable before and after transformation. The TFBFOC variable now very much aligns with the normal line.

\section{Figure 8. QQPlot of TFBFOC before and after transformation}

Table 3 provides details as to the skewness and kurtosis values associated with each of the nine variables before and after the application of the nine optimal exponents.

Table 3. Normality Before and After Box-Cox Transformation

\begin{tabular}{lccccc}
\hline & \multicolumn{2}{c}{ Skewness } & & \multicolumn{2}{c}{ Kurtosis } \\
\cline { 2 - 3 } \cline { 5 - 6 } Variable & Before & After & & Before & After \\
\hline NetUse & 1.03 & -0.14 & & 1.25 & 0.34 \\
TFBF & 1.13 & 0.05 & & 1.68 & -0.18 \\
TAFBF & 1.39 & 0.17 & & 1.63 & -0.89 \\
TFBFOC & 1.70 & 0.40 & & 3.35 & -0.30
\end{tabular}

This article is protected by copyright. All rights reserved. 
Note. $N=958$; results to two decimal places.

For each variable, skewness and kurtosis is improved substantially. Kurtosis also appeared to improve for each variable. In terms of the results (see Table 2), we can now more confidently state that the relationship between the time students spend on the net and total, actual, and campus-related friendships is only small ( $r \geq .10 \geq .29$; Cohen, 1992). There are probably many other psychological and social factors that are related to these friendship counts!

\section{Sensitivity analysis and best practice}

Research suggests that both parametric and non-parametric tests benefit from normally distributed data (see Zimmerman, 1998 for example). However, it should be noted that altering the nature of the data changes the nature of the variables themselves. For example, although the min and max values remained the same, the means and standard deviations for TFBFOC (before) was $M=98.08$ and $S D=94.15$, and TFBFOC (after) was $M=253.23$ and 101.02. This is important to consider. Thus, best practice would have it that students dealing with non-normal data (whom likely constitute many) undertake a form sensitivity analysis whereby statistical procedures are carried out in both original and transformed data contexts. As demonstrated, where results are equivalent across data conditions, the student researcher can be more certain the findings are not a result of either raw non-normal or transformed data conditions.

\section{Caveats}

It should be mentioned that the online Shiny app is not an anomaly filter and onus is placed on the student to carry out due diligence, such as checking for extreme outliers and other data aberrations prior to making use of this tool. It should also be noted that, ideally, users should make use of the app and package only after demonstrating their understanding and ability to carry out the steps as detailed in the earlier section of this paper. Use of the package or shiny app could be used as a means of confirming that the student carried out these steps on their own data correctly.

\section{Shiny hosting}

The Shiny app is hosted by local servers operated and maintained by The University of Auckland team. The authors would like to thank the ongoing support of the web-hosting team in collaboration of this project.

\section{References}

Box, G. E. P., \& Cox, D. R. (1964). An analysis of transformations. Journal of the Royal Statistical Society, B26, 211-234. Retrieved from http://www.jstor.org/discover/10.2307/2984418?uid=3738776\&uid=2\&uid=4\&sid=2110 $\underline{3336557207}$ 
Chang, K. C., \& Courtney, M. G. R. (2017). normalr: normalisation of multiple variables in large-scale datasets. R package version 0.0.2. https://github.com/kcha193/normalr

Cohen, J. (1992). A power primer. Psychological Bulletin, 112, 155-159. doi:10.1037/00332909.112.1.155

Hair, J. F., Black, W. C., Babin, B. J. and Anderson, R. E. (2010). Multivariate Data Analysis. 7th Edition. Pearson: New York.

Kowalski, C. (1972). On the Effects of Non-Normality on the Distribution of the Sample Product-Moment Correlation Coefficient. Journal of the Royal Statistical Society. Series C (Applied Statistics), 21(1), 1-12. doi:10.2307/2346598

Osborne, J. (2010). Improving your data transformations: Applying the Box-Cox transformation. Practical Assessment, Research \& Evaluation, 15(12). Retrieved from http://pareonline.net/pdf/v15n12.pdf

R Core Team (2013). R: A language and environment for statistical computing. R Foundation for Statistical Computing, Vienna, Austria. URL http://www.R-project.org/

Student (1927). Errors of Routine Analysis. Biometrika, 19(1/2), 151-164. doi. 10.2307/2332181

Zimmerman, D. W. (1998). Invalidation of parametric and nonparametric statistical tests by concurrent violation of two assumptions. The Journal of experimental education, 67(1), 55-68.

Venables, W. N., \& Ripley, B. D. (2002). Modern Applied Statistics with S (4 ${ }^{\text {th }}$ Ed.). Springer, New York. ISB 0-387-95457-0 


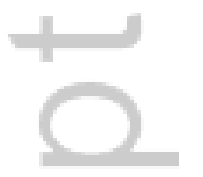

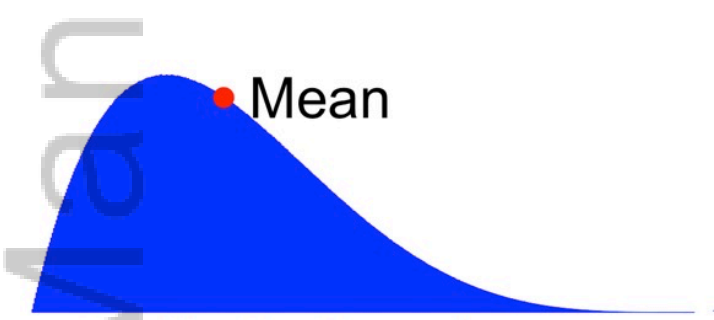

Positively-skewed

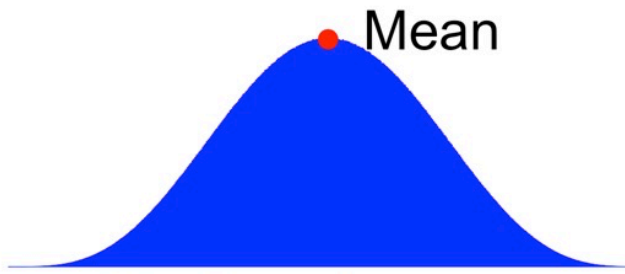

Not skewed

TEST_12154_F1.tiff
Mean

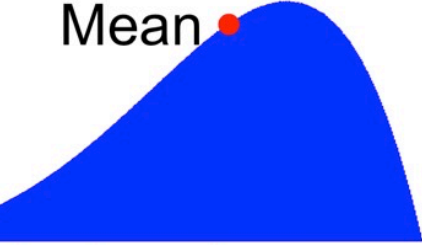

Negatively-skewed

This article is protected by copyright. All rights reserved. 


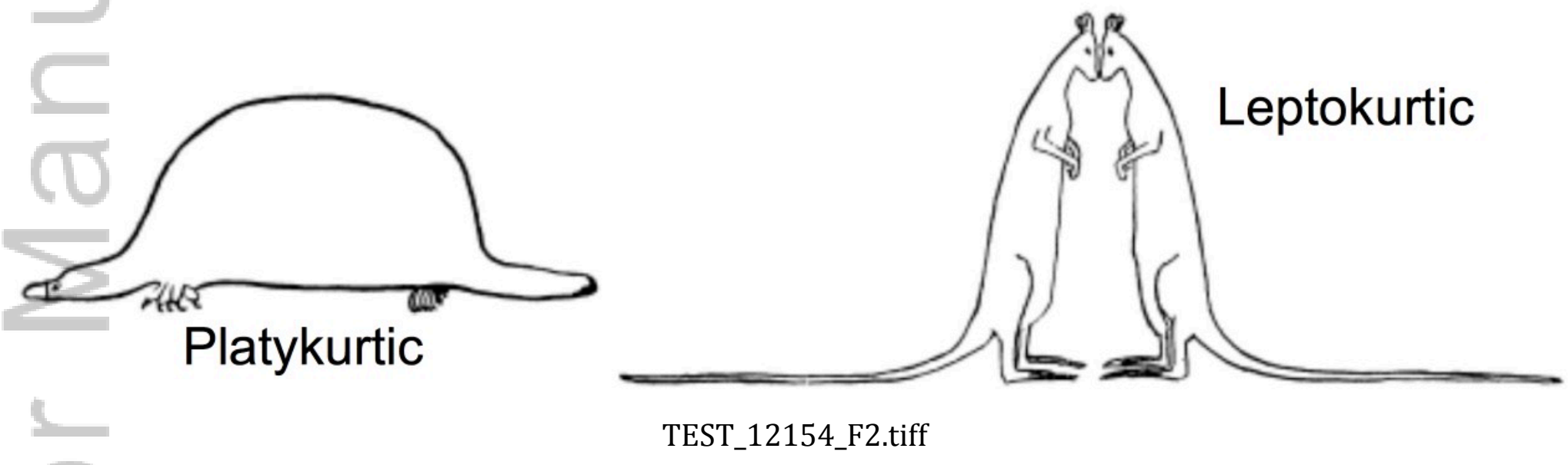

This article is protected by copyright. All rights reserved. 
Normal Q-Q Plot

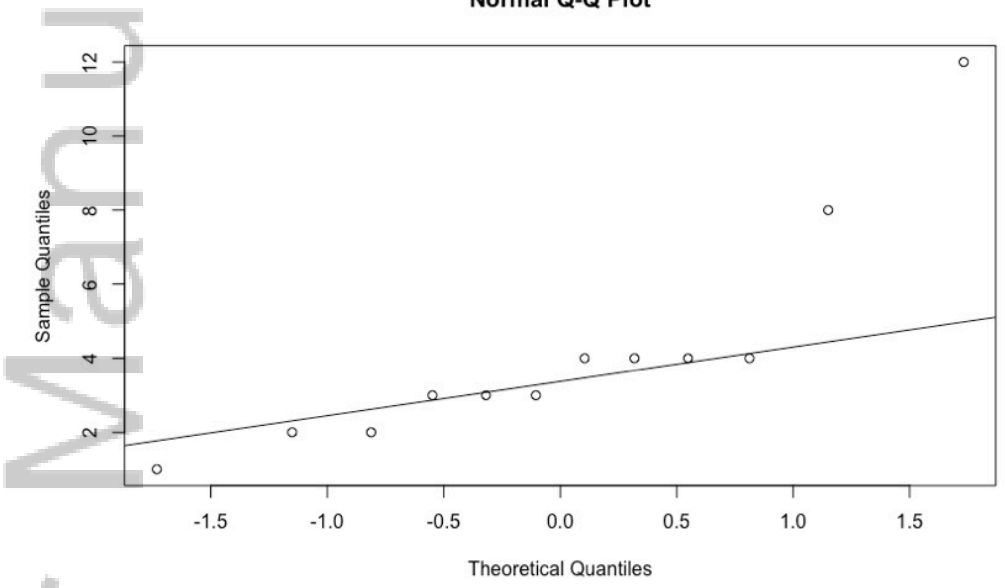

variable2

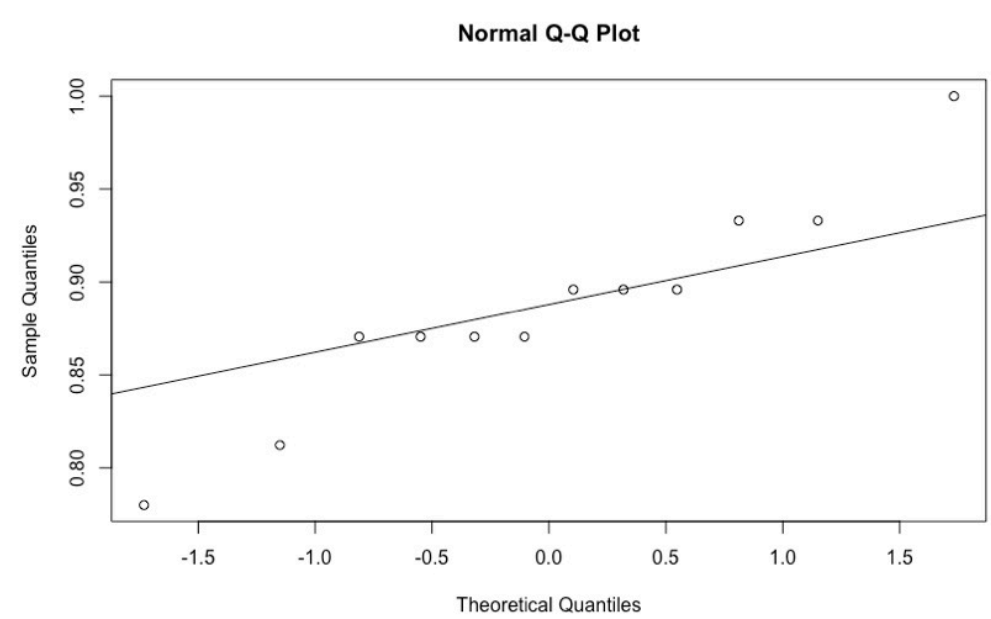

variable2.ExpNeg0 0.1

TEST_12154_F4.tiff

This article is protected by copyright. All rights reserved. 


\section{normalr $\mathrm{R}$ package}

Use test Dataset

\section{Choose CSV File}

\begin{tabular}{|l|l}
\hline Browse... Net.Habits.csv & N
\end{tabular}

\section{Upload complete}

$\checkmark$ Header

Separator

Comma

Semicolon

Tab

t. Download

Latest Update:

2017-01-17

Contact email:

k.chang@auckland.ac.nz

\section{Lambdas:}

\begin{tabular}{rrrr} 
NetUse & TFBF & TAFBF & TFBFOC \\
\hline 0.31 & 0.52 & 0.28 & 0.25
\end{tabular}

\section{Input Data:}

Original Data $\quad$ Transformed Data

\begin{tabular}{rrrr} 
NetUse & TFBF & TAFBF & TFBFOC \\
\hline 18.00 & 135.00 & 16 & 14.00 \\
\hline 8.00 & 300.00 & 10 & 30.00 \\
\hline 4.00 & 800.00 & 20 & 10.00 \\
\hline 3.00 & 50.00 & 0 & 0.00 \\
\hline 3.00 & 500.00 & 20 & 0.00 \\
\hline 3.00 & 30.00 & 10 & 5.00 \\
\hline 2.00 & 150.00 & 30 & 15.00 \\
\hline 2.00 & 215.00 & 3 & 0.00 \\
\hline 18.00 & 135.00 & 16 & 14.00 \\
\hline
\end{tabular}

TEST_12154_F6.tiff 


\section{normalr $\mathrm{R}$ package}

Use test Dataset

\section{Choose CSV File}

\begin{tabular}{l|l} 
Browse... Net.Habits.csv & N
\end{tabular}

\section{Upload complete}

$\checkmark$ Header

Separator

C Comma

Semicolon

Tab

ఓ. Download

Latest Update:

2017-01-17

Contact email:

k.chang@auckland.ac.nz

\section{Lambdas:}

\begin{tabular}{rrrr} 
NetUse & TFBF & TAFBF & TFBFOC \\
\hline 0.31 & 0.52 & 0.28 & 0.25
\end{tabular}

\section{Input Data:}

\begin{tabular}{rrrr}
\hline Original Data & \multicolumn{3}{c}{ Transformed Data } \\
\hline NetUse & TFBF & TAFBF & TFBFOC \\
\hline 18.00 & 388.97 & 231.25 & 129.72 \\
\hline 12.63 & 651.99 & 182.80 & 182.20 \\
\hline 8.99 & 1166.97 & 256.99 & 110.04 \\
\hline 7.70 & 182.84 & 0.00 & 0.00 \\
\hline 7.70 & 887.48 & 256.99 & 0.00 \\
\hline 7.70 & 111.73 & 182.80 & 75.73 \\
\hline 6.06 & 417.75 & 308.61 & 134.01 \\
\hline 6.06 & 528.87 & 90.59 & 0.00 \\
\hline 18.00 & 388.97 & 231.25 & 129.72 \\
\hline
\end{tabular}

TEST_12154_F7.tiff

This article is protected by copyright. All rights reserved. 


\section{University Library}

\section{- M M I N E R VA A gateway to Melbourne's research publications}

Minerva Access is the Institutional Repository of The University of Melbourne

Author/s:

Courtney, MGR;Chang, KC

Title:

Dealing with non-normality: an introduction and step-by-step guide using $R$

Date:

2018-06-01

Citation:

Courtney, M. G. R. \& Chang, K. C. (2018). Dealing with non-normality: an introduction and step-by-step guide using R. TEACHING STATISTICS, 40 (2), pp.51-59. https:// doi.org/10.1111/test.12154.

Persistent Link:

http://hdl.handle.net/11343/283879 